\title{
Surfactant Induced Photostability Enhancements of Thiol Coated Quantum Dot Nanocolloids
}

\author{
Abu Mohammad Azmal Morshed and Tae Hyun Yoon \\ Nanoscale Characterization and Enwironmental Chemistry Laboratory, Deparment of Chemistry, Hamvang Lniversity, \\ Seoul 133-791, Korea. ${ }^{*}$ E-mail: taevoonahanvang.ackr \\ Received October 25, 2007
}

Key Words : Photostability. Environmental impact of nanoparticles. Nanotoxicology

Over the past decades, nanosized inorganic particles, such as nano- $\mathrm{TiO}_{-}$, nano- $\mathrm{Au}$, nano-Ag and seniconductor quantum dots (QDs) have attracted great attentions from many areas of sciences and engineerings. due to their unique quantum size effect as well as their huge potentials in many areas of applications (e.g., display devices, sensors. solar cells. molecular intaging probes etc.). However. mass production and extensive use of these nanomaterials raise increasing concerns about their potential adverse effect on our environment and human health. when they are accidentally released into our environments. Therefore. chemical speciation and toxicity studies of these nanoparticles are required to minimize their potential adverse effects as well as further advancements of nanotecluology and related industries.

Among these engineered nanomaterials. aqueous colloidal QDs (e.g. CdS. CdSe, $\mathrm{PbS}$ etc.) have great potential as a new type of fluorophore for the biological and medical imaging. ${ }^{1-4}$ Because aqueous solubility is one of the most important requirements for the biological and medical applications. various methods for the preparation of watersoluble QDs have been developed recently, such as surface modifications with amphiphilic molecules including thiol containing compounds and phospholipids." Among these strategies to suspend QDs in aqueous solution, ligand exchange reaction with thiol containing compounds. i.e.. mercapto carbonic acid. has been recognized as one of the standard methods and generally used for aqueous solubilization of QDs. However, these ligand exchange reactions typically modify physicochemical states of the QD surface atoms and dramatically change various properties of QDs. including the photostability of QD surface atoms, resistance to oxidative dissolution process and coagulation/precipitation tendency. For instance, it has been suggested that thiol groups are easily photooxidized to form disulfides with neighboring thiol molecules and the resultant hydrophilic disulfide molecules can readily removed from the QD surface and dissolved into aqueous solution, which cause aggregation and precipitation of QDs. ${ }^{6}$ Moreover. it has been suggested that the oxygen molecules in air also participate in the oxidation of Se atoms located at the surface of QDs and the resultant $\mathrm{SeO}_{3}{ }^{2-}$ and $\mathrm{Cd}^{\hat{2}^{-}}$ion species desorbed from the surface. Therefore, long UV exposure of thiol coated QDs under oxidative environment may induce decomposition of the QD nanocrystals and subsequently cause the release of toxic $\mathrm{Cd}^{++}$ions. ${ }^{7}$ However. despite of its potential significance as a new pollutant and toxic materials. only limited number of studies have been performed on the photostability of these nanomaterials under relevant environmental condition yet.

In this study. we have investigated the interactions of QD surface functionalities with various artificial surfactant molecules and their impacts on the photostabilities of QDs. It is based on the assumption that surfactant molecules $(e . g$., sodium dodecyl sulfate (SDS), sodium dodecyl benzene sulfonate (SDBS) and cetyl trimethyl ammonium bromide (CTAB)) added to the thiol coated QDs may have stabilizing effect on nanocolloids as well as reduction/enhancement of the surface oxidation via formations of micelles or vesicles.

Photographs of QD solutions at different time intervals are presented in Figure l(a). At the begining of the UV exposure experiments. aqueous suspensions of four different QDs exhibited the same visible colors with each other. However, after long exposure to UV radiation. distinct visual color changes were clearly observed. indicating different photostability of these aqueous suspended QDs. Obviously, QDs coated with MAA only precipitated much faster than the QD-MAA's associated with surfactants. Of particular importance. QD-MAA associated with cationic CTAB surfactant seems the most resistant to the coagulation and precipitation processes, confirmed by the orange colored QD solution observed even after 150 hours of UV exposure experiment.

To obtain further information on these colloidal properties. we collected UV-visible spectra of each QD solutions at different time interval and further processed to extract detailed spectral features, such as position, width and height of the first exciton absorption of QDs (see Fig. l(b) for the full spectral set of QD-MAA-CTAB solution). Results from UV/ris spectral analysis for different QDs were also presented in Figure 2. As previously reported. ${ }^{9}$ the peak position and intensity of the furst exciton absorption of QD nanocrystals are good indicator of $\mathrm{CdSe}$ core size and concentration of suspended QDs. Moreover. the high absorption coefficients of QD nanocrystals $\left(\sim 10^{5} \mathrm{~cm}^{-1} \mathrm{M}^{-1}\right)^{\vartheta}$ made conventional UV/ris spectroscopy as a very sensitive probe for quantitative monitoring of diluted aqueous QDs solutions even in the range of nanomolar concentrations. 

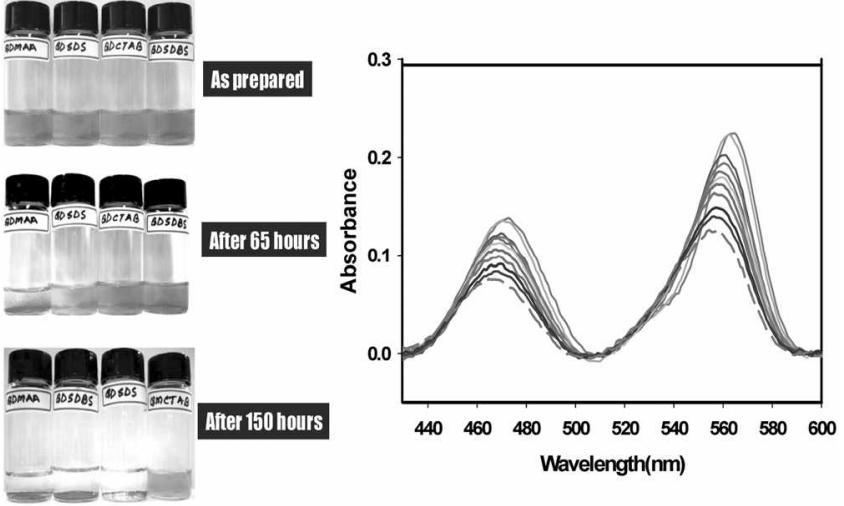

Figure 1. (a) Photographs of the QD-MAA suspensions $w /$ or $w / 0$ various surfactants (QD-MAA, QD-MAA-SDS, QD-MAA-SDBS and QD-MAA-CIAB from left to right) (b) Baseline subtracted $U V / V i s$ spectral sets of the QD-MAA-C'IAB ayueous suspension collected at different time intervals.
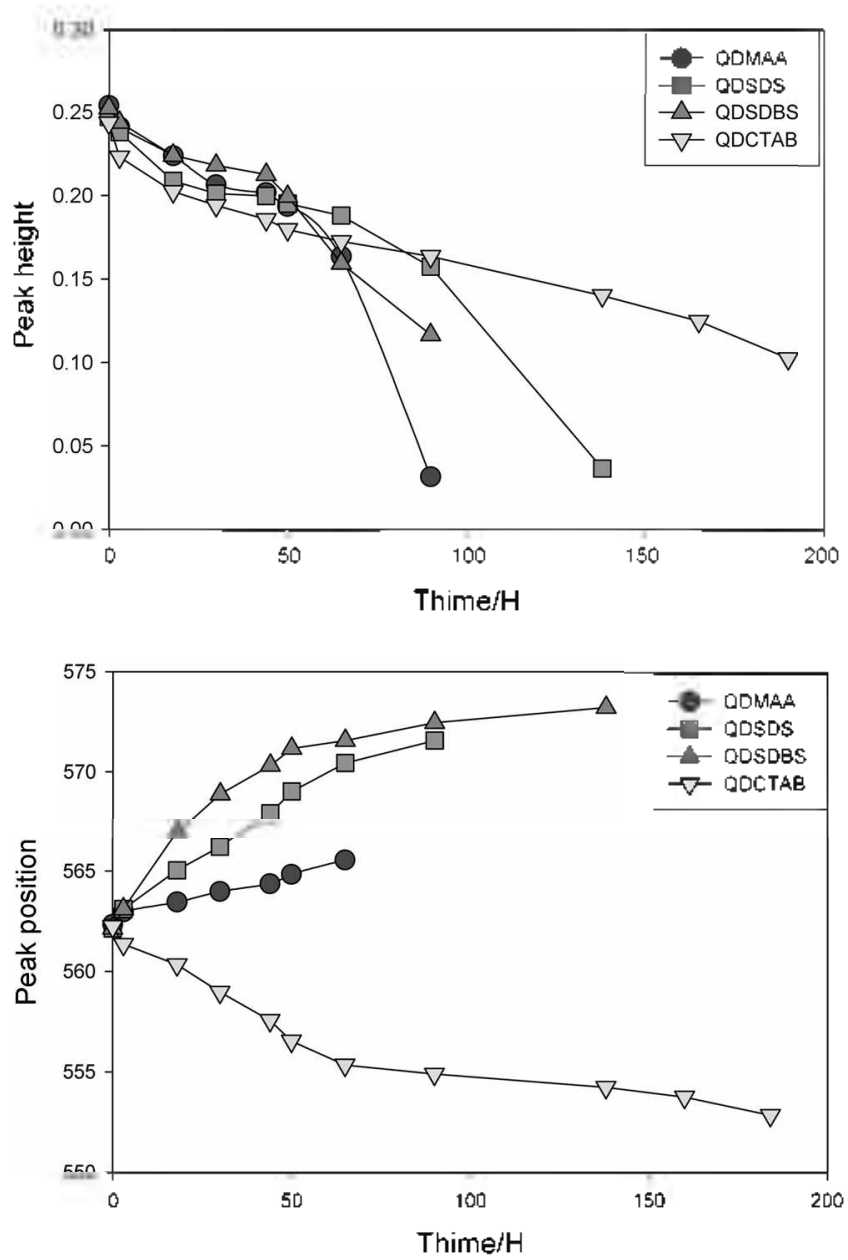

Figure 2. Temporal evolution of (a) peak heights and (b) peak positions of the first exciton absorption of QD suspensions

As can be seen in Figure l(b) and Figure 2, significant temporal changes in the peak position. width and height were observed. For instance. in the case of QD-MAA-CTAB shown in Figure 1(b). the peak position of the first exciton absorption peak progressively moved to lower wavelength
$(562 \mathrm{~nm} \rightarrow 555 \mathrm{~mm}$, blue shifted $7 \mathrm{~lm}$ durng the first 60 hours of UV exposure) then slowed down in its blue-shift rate (only $2 \mathrm{~nm}$ further blue-shift of the first exciton absorption peak position during $60-170$ hours period). Blue-shift of the first exciton absorption peak position indicates decrease in the QD nanoparticle diameter ( $3.31 \mathrm{~lm} \rightarrow 3.15 \mathrm{~nm}$. based on the empirical equation proposed by $\mathrm{Yu} e t a l^{9}$ ), due to oxidative dissolution of QDs under given environment of long UV radiation exposure. In addition, reduced optical density (OD) at the first exciton absorption peak of QDs imply precipitation process occurring under given environments. while the increase of peak width indicates broadening of this QD particle size distributions. probably due to the different rate of dissolution and ripening processes simultaneously occurring under given condition. Therefore, from the temporal evolution of the UV/vis spectral features. it was found that QD-MAA-CTAB nanoparticles experience significant decrease in their particle size accompanied by very slow aggregation/precipitation of QD particles.

Comparisons of the temporal peak height changes for the four different QD suspensions (shown in Fig. 2(a)) provide information on the relative photostabilities among these QDMAA's w/ or w/o surfactants. QD-MAA w/o surfactants precipitated out of the solution most readily. while QDMAA-CTAB forms the most stable suspension among QD suspensions. For the surfactants used in this study. i.e., SDS, SDBS and CTAB, it seems that they all have stabilizing effect for thiol capped QD nanocrystals and the photostability of the dispersed QDs are in the order of QD-MAA $<$ QD-MAA-SDS < QD-MAA-SDBS < QD-MAA-CTAB We also found that cationic surfactant. such as CTAB, is more efficient than anionic counterparts in stabilizing thiol capped QDs. which is negatively charged in neutral $\mathrm{pH}$ conditions.

Temporal evolution of the peak positions shown in Figure 2(b) also provide interesting trends among QD-MAA's with different stabilizing surfactants. In contrary to the "blue" shift observed for QD-MAA-CTAB, the other QD-MAA's with or without surfactant molecules showed peak shifts to the higher wavelengths. (i.e.. "red" shift), implying increase in their QD particle sizes (i.e.. ripening or coagulation of the QD particles). In addition. continuous broadening in the peak widths (i.e., broadening of the QD particle size distribution) were also observed for each QD suspensions. implying that oxidative dissolution and coagulation/ripening process were occurring simultaneously under given UV exposure condition and the direction of the peak shifts (i.e. blue or red) depends on the dominating process occurring under given environment.

Further consideration of QD photooxidation mechanisms and the molecular differences among surfactants may provide an insight to interprete our observations. As briefly mentioned before. upon absorption of the UV light by QDs. absorbed energies will be transferred to the surface of the QDs and induce photooxidation of surface thiol groups to form disulfide molecules. which is water-soluble and readily removed from the QD surface and dissolved into aqueous 
solution. As a result hydrophobic surfaces of the QDs will be exposed to aqueous environment and these partially naked QD surfaces will prefer to stick together to form aggregates of QDs and cause increases in their core size (i.e.. "red" shift in UV/vis absorption peak position). On the other hand, the oxidative dissolution of the QD surface can also occur simultaneously as a result of the transfer of absorbed energy to the surface of QDs and following oxidation of surface $\mathrm{Se}$ atoms. The resultant Se species $\left(e . g . \mathrm{SeO}_{3}{ }^{{ }^{-}-}\right)$and $\mathrm{Cd}^{\hat{3}}$ ion will be desorbed from the QD surface and cause "blue" shift of the first exciton absorption peak of QDs (i.e. the reduction in their core size).

These processes are probably the two main reaction pathways occurring at the QD surface under given experimental condition of UV radiation. We think that the presence of different surfactant molecules will affect (inhibiting or enhancing) relative contribution of these processes by forming different type of micelles/vesicles. For instance. $\mathrm{CTAB}$ is a cationic surfactant and can readily form vesicles or micelles around the negatively charged QD-MAA surface through electrostatic interactions. These well-assembled vesicles or micelles of CTAB will act as a strong diffusion barrier for the partially naked QDs to transport and encounter with other QDs. as a result, preventing them from coagulation and precipitation. This characteristic of CTAB seems to be the main reason for the slower coagulation/ ripening process of QDs and limited contribution of "red" shift observed for QD-MAA-CTAB. while oxidative dissolution of QDs are still in progress within the CTAB vesicles or micelles, which cause observed "blue" shift of QDs under the UV radiation. Moreover, CTAB will also prevent removal of water-soluble disulfides from the $\mathrm{QD}$ surface and cause longer stability of QD-MAA nanoparticles in aqueous solution. which result in much slower decay of UV/vis peak intensities. In contrast. anionic surfactants. such as SDS and SDBS. will have repulsive electrostatic interactions with negatively charged QD-MAA's and may not be able to form stable vesicles or micelles around the QDs. Therefore. these anionic surfactant can not act as a strong diffusion barrier for the partially naked QDs and facilitate easy' transport of the QDs out of the SDS (or SDBS) vesicles or micelles so that they can encounter other QDs to form QD aggregates. which will induce further coagulation process dominating over oxidative dissolution process.

Therefore from our observations. we propose that the type and characteristics of the surfactant used for stabilizing QDs have significant roles in determining reaction pathway's of QD colloidal particles under the UV radiation. We believe that our observations may provide important clues on the role of various surfactants. weather synthetic or natural. on the colloidal stability of various nanoparticles in aqueous solutions. which is particularly important for the toxi- cological study of these nanomaterials as well as the medical and biological applications of these nanoparticles. Further studies are also in progress in our group to systematically understand these phenomena caused by synthetic or natural surfactant molecules.

\section{Experimental section}

Sodium dodecyl sulfate (SDS). Cetyltrimethylanmonium bromide (CTAB), Sodiumdodecylbebzene sulfunate (SDBS) were above $99 \%$ purity and purchased from Sigma. Marceptoacetic acid (MAA) is from TCI, Japan $(99 \%)$. All other chemicals used in this study were above reagent grade and directly used without further purification. TOPO capped QDs were obtained from Nanosquare Co. Ltd.. which were synthesized using a SiPOP procedure. ${ }^{8}$ This TOPO capped QDs were solubilized in aqueous solution wa ligand exchange reaction with MAA and then further modified with various surfactants such as SDS. SDBS and CTAB. All UV exposure experiments were performed in a photooxidation chamber under constant airflow equipped with a UV lamp (peak maximum at $300 \mathrm{rm}$ ), which were placed $\sim 4 \mathrm{~cm}$ directly from the samples. The absorption spectra were recorded at certain time intervals with UV/VIS Spectrophotometer (OPTIZEN 2120UV MECASYS). Concentrations of QDs in aqueous solutions were determined from the intensities of the first excitonic absorption peak and extinction coefficients based on the method reported by Yu $e t a f^{5}$ and presented in the units of moles of QD per liter (i.e. [QD]). Peak position, height and width of the first exciton absorption of QD nanoparticles were used to monitor the physical changes of QD particles. i.e.: average diameter of QDs. estimated amount of suspended QDs and heterogeneity of $\mathrm{QD}$ particle size distributions

Acknowledgements. This work was supported by the research fund of Hanyang University (HY-2005-N). We also thanks to Nanosquare Co. Ltd. for providing quantum dot raw materials used for this study.

\section{References}

1. Bruchez, M. Jr.: Moronne. M.; Gin. P.: Weiss. S.: Alivisatos. A. P. Science 1998. 281, 2013

2. Chan. W. C. W. Nie. S. Science 1998. 281.2016.

3. Hwang. C.-S.: Cho. I.-H. Bull. Korean Chen. Soc. 2005. 26. 1776

4. Lee. H. T.: Kim. D.-Y.: Yoo. I.-S.: Bang. J.: Kim. S.: Park. S. M. Bull. Korean Chem. Soc. 2007, 28.953.

5. Yu. W. W.: Chang, E.; Drezek, R.: Colvin. V. L. Biochem. Biophy Res. Comm. 2006, 348.781

6. Aldana. J.: Wang. Y. A.: Peng. X. G. J. Ant Chem. Soc. 2001 . 123. 8844.

7. Derfus. A. M.: Chan. W. C.: Bhatia. S. Namo Lett. 2004. f. 11

8. Kim. J. I.: Lee. J. K. Adv Func. Haten 2006. 16, 2077.

9. Yu. W. W.: Qu. L: Guo, W.: Peng, X. Chem Hater: 2003. 15. 2854. 